\title{
X-ray reflection spectroscopy with Kaluza-Klein black holes
}

\author{
Jiachen Zhu ${ }^{1}$, Askar B. Abdikamalov ${ }^{1}$, Dimitry Ayzenberg ${ }^{1}$, Mustapha Azreg-Aïnou ${ }^{2}$, Cosimo Bambi ${ }^{1, a}$, \\ Mubasher Jamil $^{3,4,5}$, Sourabh Nampalliwar ${ }^{6}$, Ashutosh Tripathi ${ }^{1}$, Menglei Zhou ${ }^{1}$ \\ ${ }^{1}$ Center for Field Theory and Particle Physics, Department of Physics, Fudan University, Shanghai 200438, China \\ ${ }^{2}$ Engineering Faculty, Baskent University, 06790 Ankara, Turkey \\ ${ }^{3}$ Institute for Theoretical Physics and Cosmology, Zhejiang University of Technology, Hangzhou 310023, China \\ ${ }^{4}$ Department of Mathematics, School of Natural Sciences (SNS), National University of Sciences and Technology (NUST), H-12, Islamabad \\ 44000, Pakistan \\ ${ }^{5}$ Canadian Quantum Research Center, 204-3002 32 Ave, Vernon, BC V1T 2L7, Canada \\ 6 Theoretical Astrophysics, Eberhard-Karls Universität Tübingen, 72076 Tübingen, Germany
}

Received: 20 April 2020 / Accepted: 2 July 2020 / Published online: 14 July 2020

(C) The Author(s) 2020

\begin{abstract}
Kaluza-Klein theory is a popular alternative theory of gravity, with both non-rotating and rotating black hole solutions known. This allows for the possibility that the theory could be observationally tested. We present a model which calculates the reflection spectrum of a black hole accretion disk system, where the black hole is described by a rotating solution of the Kaluza-Klein theory. We also use this model to analyze X-ray data from the stella-mass black hole in GRS 1915+105 and provide constraints on the free parameters of the Kaluza-Klein black holes.
\end{abstract}

\section{Introduction}

General relativity, the standard theory of gravitation today, has been applied to a large range of astrophysical phenomena in our Universe. Over the years, it has undergone a plethora of tests; while largely successful in the weak-field regime [1], its predictions in the strong-field regime have recently become testable in a variety of ways [2-4]. During this period, various shortcomings of general relativity (GR hereafter), both from the theoretical (e.g, singularities, difficulty integrating with quantum mechanics, the hierarchy problem) as well as observational point of view (e.g., dark matter, dark energy), have been exposed. Resolutions range from conservative (additional fields, extensions with GR as a limit) to radical (modifications to GR) proposals. In either case, without testable predictions it is impossible to determine if the resolution, conservative or radical, is valid.

Black holes (BHs hereafter) promise to be the strongest probes of gravity in our Universe. Their compactness means

\footnotetext{
a e-mail: bambi@ fudan.edu.cn (corresponding author)
}

gravitational effects, of GR or of alternatives to GR, are strongest in their vicinity. Their simplicity within GR means deviations away from GR, if imprinted on BH solutions, could be easily detected. Finally, their ubiquitousness in nature means there are several potential sources to study. Thus, testing alternative theories of gravity is particularly promising with BHs.

Gravitational waves, imaging of BH shadow, and X-ray spectroscopy are the leading techniques for probing astrophysical BHs. Among these, imaging is not expected to provide very strong constraints on alternative theories [5]. Gravitational wave interferometry is the most promising technique, though in some cases, it is expected to be comparable to $\mathrm{X}$-ray spectroscopy [6]. ${ }^{1}$ In the present work, our focus is on the X-ray spectroscopy technique. Specifically, we are interested in the reflection spectrum of $\mathrm{BH}$ accretion disks $[2,9,10]$. For Kerr BHs of GR, RELXILL is the leading model for analysis of the reflection spectrum [11,12]. Some of us have been involved in generalizing this model to non-Kerr metrics [13,14]. The model, RELXILL_NK, has been applied to X-ray observations of several astrophysical BHs to place constraints on deviations away from the Kerr solution [1528]. A public version of the model is available at $[29,30]$. Another interesting phenomenon that can potentially be used to probe strong-field gravity is the gyroscope precession frequency. The gravitational field of the $\mathrm{BH}$ causes the rotational axis of a gyroscope to precess, and the precession frequency carries unique signatures of the BH metric. Such precession frequencies have been calculated in several non-Kerr spacetimes [31-34].

\footnotetext{
${ }^{1}$ In some other cases, e.g. violations of the Einstein Equivalence Principle like variation of fundamental constants [7] or deviations from geodesic motion [8], it will be unable to provide any constraint.
} 
One of the most interesting alternative theory of gravity proposals is the Kaluza-Klein theory. With purely classical origins back in 1919, the theory has been interpreted in a quantum mechanical as well as a string theory framework. It is a five-dimensional theory, with a compact fifth dimension. The basic ingredients include three kinds of fields: gravity, electromagnetism and a scalar field. Tests of the theory include looking for signatures in the Large Hadron Collider [35], but such tests have not been very successful yet. Among astrophysical tests, in [36] the authors have analyzed how equations of motion change in Kaluza-Klein cosmology which may affect motions of galaxies. Shadows of KaluzaKlein BHs have also been analyzed in [37]. Gravitational waves are not expected to provide good constraints in the near future [38] (See also [39]). In [40], some of us study the precession of a gyroscope in the vicinity of a Kaluza-Klein $\mathrm{BH}$. We can therefore ask the question: can the predictions of Kaluza-Klein theory be tested using X-ray spectroscopy? This paper presents our efforts to answer this question.

BHs in Kaluza-Klein theory have been derived in various limits [41]. Non-rotating spherically symmetric BHs were derived in [42-44]. Larsen (among others) found rotating $\mathrm{BH}$ solutions in five and four dimensions [45-47]. BHs with squashed horizon were calculated in [48,49]. Six and higher dimension versions have also been found [50]. Since astrophysical BHs are mostly rotating, and X-ray spectroscopy is most suited for rapidly rotating BHs, we shall focus on rotating BHs. Rotating Kaluza-Klein BHs typically have four free parameters: mass, spin and the electric and magnetic charges. We have implemented this BH metric in the RELXILL_NK framework, and used data from an X-ray binary to get constraints on the free parameters.

The paper is organized as follows: in Sect. 2, we review the Kaluza-Klein theory and the BH metric. In Sect. 3 we review the theory of X-ray spectroscopy, the RELXILL_NK framework, and describe the numerical method we used to implement the Kaluza-Klein BH metric in RELXILL_NK. In Sect. 4, the new model is applied to X-ray observations of an $\mathrm{X}$-ray binary. Conclusions follow in Sect. 5.

\section{The metric}

We will follow the notation developed in [40] for the metric. The simplest Kaluza-Klein theory involves three fields: gravity, the dilaton and the gauge field. The action in the Einstein frame is [51]:

$S=\int \sqrt{-g}\left(\frac{R}{\kappa^{2}}+\frac{1}{4} e^{\sqrt{3} \kappa \sigma} F_{\alpha \beta} F^{\alpha \beta}+\frac{1}{2} \nabla^{\alpha} \sigma \nabla_{\alpha} \sigma\right) d^{4} x$,

where $\sqrt{-g}$ is the determinant of the four-dimensional metric tensor, $R$ the Ricci scalar, $F_{\alpha \beta}$ the gauge field which can be identified with the electromagnetic field, and $\sigma$ a dilaton scalar field. $\kappa$ is a constant and is equal to $\sqrt{16 \pi G}$.

Although we will not derive the $\mathrm{BH}$ solution here, it is interesting to point out some features of the solution generating techniques. Standard methods of solving the field equations can be used to derive the non-rotating solutions. Rotating solutions on the other hand have been obtained in the following ways: for slow rotation, [52] solve the complete field equations perturbatively, following [53]; others [41,45] boost the Kerr metric along a line to get a five-dimensional rotating $\mathrm{BH}$ solution. The metric looks like this:

$$
\begin{aligned}
\mathrm{d} s^{2}= & \frac{H_{2}}{H_{1}}(\mathrm{~d} \psi+A)^{2}-\frac{H_{3}}{H_{2}}(\mathrm{~d} t+B)^{2} \\
& +H_{1}\left(\frac{\mathrm{d} r^{2}}{\Delta}+\mathrm{d} \theta^{2}+\frac{\Delta}{H_{3}} \sin ^{2} \theta \mathrm{d} \phi^{2}\right),
\end{aligned}
$$

where the one-forms are given by

$$
\begin{aligned}
A= & -\frac{1}{H_{2}}\left[2 Q\left(r+\frac{p-2 m}{2}\right)+\sqrt{\frac{q^{3}\left(p^{2}-4 m^{2}\right)}{4 m^{2}(p+q)}} a \cos \theta\right] \mathrm{d} t \\
& -\frac{1}{H_{2}}\left[2 p\left(H_{2}+a^{2} \sin ^{2} \theta\right) \cos \theta\right. \\
& +\sqrt{\frac{p\left(q^{2}-4 m^{2}\right)}{4 m^{2}(p+q)^{3}}}[(p+q)(p r-m(p-2 m)) \\
& \left.\left.+q\left(p^{2}-4 m^{2}\right)\right] a \sin ^{2} \theta\right] \mathrm{d} \phi \\
B= & \frac{\left(p q+4 m^{2}\right) r-m(p-2 m)(q-2 m)}{2 m(p+q) H_{3}} \sqrt{p q} a \sin ^{2} \theta \mathrm{d} \phi
\end{aligned}
$$

and

$$
\begin{aligned}
H_{1} & =r^{2}+a^{2} \cos ^{2} \theta+r(p-2 m)+\frac{p(p-2 m)(q-2 m)}{2(p+q)} \\
& -\frac{p}{2 m(p+q)} \sqrt{\left(q^{2}-4 m^{2}\right)\left(p^{2}-4 m^{2}\right)} a \cos \theta, \\
H_{2} & =r^{2}+a^{2} \cos ^{2} \theta+r(q-2 m)+\frac{q(p-2 m)(q-2 m)}{2(p+q)} \\
& +\frac{q}{2 m(p+q)} \sqrt{\left(q^{2}-4 m^{2}\right)\left(p^{2}-4 m^{2}\right)} a \cos \theta, \\
H_{3} & =r^{2}+a^{2} \cos ^{2} \theta-2 m r, \\
\Delta & =r^{2}+a^{2}-2 m r .
\end{aligned}
$$

The solution admits four free parameters, viz. $m, a, p, q$, which are related to the physical mass $M$, the angular momentum $J$ and the electric $(Q)$ and magnetic charge $(P)$ respectively. The relations are given as: 


$$
\begin{aligned}
M & =\frac{p+q}{4}, \\
J & =\frac{\sqrt{p q}\left(p q+4 m^{2}\right)}{4 m(p+q)} a, \\
Q^{2} & =\frac{q\left(q^{2}-4 m^{2}\right)}{4(p+q)}, \\
P^{2} & =\frac{p\left(p^{2}-4 m^{2}\right)}{4(p+q)} .
\end{aligned}
$$

The fifth dimension can be compactified and this results in a four-dimensional BH metric [40]:

$$
\begin{aligned}
d s^{2}= & -\frac{H_{3}}{\rho^{2}} \mathrm{~d} t^{2}-2 \frac{H_{4}}{\rho^{2}} \mathrm{~d} t \mathrm{~d} \phi+\frac{\rho^{2}}{\Delta} \mathrm{d} r^{2}+\rho^{2} \mathrm{~d} \theta^{2} \\
& +\left(\frac{-H_{4}^{2}+\rho^{4} \Delta \sin ^{2} \theta}{\rho^{2} H_{3}}\right) \mathrm{d} \phi^{2}
\end{aligned}
$$

where $\rho=\sqrt{H_{1} H_{2}}$ and

$$
\begin{aligned}
\frac{H_{1}}{M^{2}} & =\frac{8(b-2)(c-2) b}{(b+c)^{3}}+\frac{4(b-2) x}{b+c}+x^{2} \\
& -\frac{2 b \sqrt{\left(b^{2}-4\right)\left(c^{2}-4\right)} \alpha \cos \theta}{(b+c)^{2}}+\alpha^{2} \cos ^{2} \theta \\
\frac{H_{2}}{M^{2}} & =\frac{8(b-2)(c-2) c}{(b+c)^{3}}+\frac{4(c-2) x}{b+c}+x^{2}+ \\
& \frac{2 c \sqrt{\left(b^{2}-4\right)\left(c^{2}-4\right)} \alpha \cos \theta}{(b+c)^{2}}+\alpha^{2} \cos ^{2} \theta \\
\frac{H_{3}}{M^{2}} & =x^{2}+\alpha^{2} \cos ^{2} \theta-\frac{8 x}{b+c} \\
\frac{H_{4}}{M^{3}} & =\frac{2 \sqrt{b c}[(b c+4)(b+c) x-4(b-2)(c-2)] \alpha \sin ^{2} \theta}{(b+c)^{3}}, \\
\frac{\Delta}{M^{2}} & =x^{2}+\alpha^{2}-\frac{8 x}{b+c} .
\end{aligned}
$$

Here we have used dimensionless version of the free parameters, defined as $\alpha \equiv a / M, b \equiv p / m, c \equiv q / m$, and $x \equiv r / M$. Moreover, we can relate the free parameter $m$ and the physical mass $M$ using Eq. 4 and obtain

$m=4 M /(b+c)$.

Note that the spin parameter $\alpha$ is not always the same as the dimensionless spin parameter of the Kerr metric. Only when the electric and magnetic charges are zero, and the KaluzaKlein metric reduces to the Kerr metric, does $\alpha$ equal the $a_{*}$ parameter of the Kerr solution.

We now discuss some properties of this solution. It admits two horizons, viz.

$r_{ \pm}=m \pm \sqrt{m^{2}-a^{2}}$

or, in terms of the dimensionless quantities,

$$
x_{ \pm}=\frac{4 \pm \sqrt{16-\alpha^{2}(b+c)^{2}}}{b+c},
$$

and the determinant is equal to $\rho^{2} \sin ^{2} \theta$. The non-rotating class of solutions $(\alpha=0)$ was obtained in [44]. For $b=c$, the non-rotating solution reduces to the Reissner-Nordström solution of GR. The Kerr solution is recovered when $b=$ $c=2$. However, when magnetic charge is zero, the metric does not reduce to the Kerr-Newman solution. Here, we are interested in BHs that parametrically deviate from the Kerr BHs. Since our data analysis models allow only one variable deformation parameter ${ }^{2}$, we cannot allow both $b$ and $c$ to be free. Therefore, we consider the following two cases ${ }^{3}$ :

- Case $1: b=c$.

- Case 2: $b$ free, $c=2$.

Case 1 describes BHs with electric and magnetic charges; since the electric charge of macroscopic astrophysical black holes is thought to be negligible, this case should be seen as a toy-model and to illustrate the capability of X-ray reflection spectroscopy to test such a scenario with observations. Case 2 describes BHs with vanishing electric charge and nonvanishing magnetic charge; such a scenario is theoretically more motivated. Magnetically charged black holes cannot be neutralized with ordinary matter and the possibility of the existence of magnetically charged black holes can be seen as a prediction of string theory, which requires to be tested against observations [54,55].

During the analysis, it is important to ensure the spacetime does not have pathologies. Requiring that the metric structure is preserved everywhere outside the horizon leads to bounds on the free parameters. We will determine these bounds now. First, following the definition of $Q^{2}$ and $P^{2}$ in Eq. 4, we have the conditions $q \geq 2 m, p \geq 2 m$, or $b \geq 2, c \geq 2$. Using these and Eq. 9 we arrive at a bound on $\alpha$ :

$\alpha^{2}<1$, or $-1<\alpha<1$.

For upper bounds on $b$, we use Eqs. 9 and 10, and get the following inequalities for the two cases defined above:

- Case 1:

$$
2 \leq b<\frac{2}{|\alpha|} .
$$

- Case 2:

$$
2 \leq b<\frac{4}{|\alpha|}-2 .
$$

\footnotetext{
2 The primary reason for this limitation is that the size of the FITS file (see Sect. 3.3) that is loaded in the computer's RAM is 1.4 GB for one deformation parameter and $42 \mathrm{~GB}$ for two. The former is manageable, the latter is not.

${ }^{3}$ Note that the case with $b=2$ and $c$ free (BHs with non-vanishing electric charge and vanishing magnetic charge) is analogous to Case 2, since the metric in Eq. 5 has similar forms for the two choices.
} 


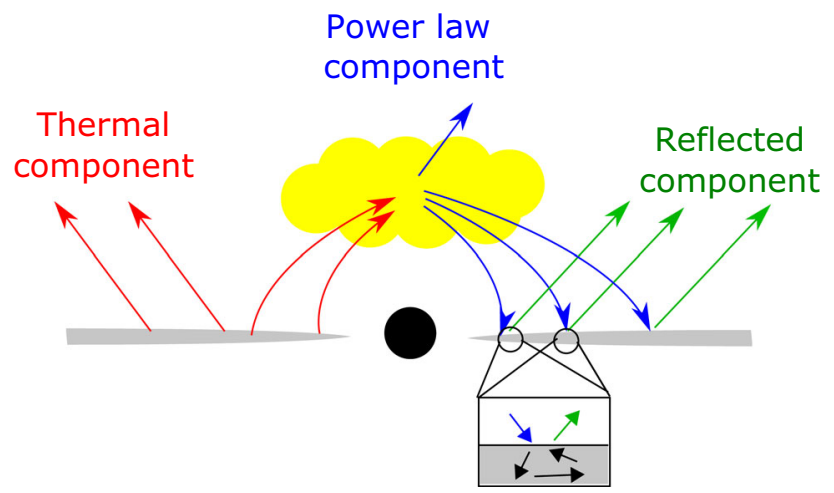

Fig. 1 A cartoon of the disk-corona model. The black circle in the center indicates the $\mathrm{BH}$, the disk is indicated in grey, and the corona by a yellow cloud. The structure of the corona is poorly understood so this illustration is only a guess. The arrows indicate photons and are colored according to the classification labeled on the figure and discussed in the text

For the allowed range of $c$, note that in Case $1 b$ equals $c$, so $c$ shares the same range as $b$, while in Case $2 c$ is fixed at 2 .

\section{X-ray reflection spectroscopy}

\subsection{Theory}

The standard astrophysical system we consider is a stationary BH surrounded by an accretion disk. The disk could be generated by a companion (as in stellar-mass X-ray binaries) or galactic material (as around active galactic nuclei). The typical model for a $\mathrm{BH}$-accretion disk system is the disk-corona model. Figure 1 shows a cartoon of this model. The BH is assumed to be surrounded by an optically thick and geometrically thin disk in the equatorial plane [56], with its inner edge at some radius $r_{\text {in }}$, bounded by the innermost stable circular orbit (ISCO hereafter), and the outer edge at a radius $r_{\text {out }}$. The system also includes a "corona". The corona is a cloud of hot plasma (effective temperature of the order of $100 \mathrm{keV}$ ) whose morphology is not understood very well. The radiation spectrum includes a power-law component, produced by inverse Compton scattering of photons from the disk by the corona, a thermal continuum of blackbody radiation from the particles in the disk, and a reflection component, produced when the upscattered photons return to the disk and are reflected after reprocessing inside the disk. Our focus in the present work is on the reflection component. It is a sensitive feature and is affected not just by the $\mathrm{BH}$ (driving the photon from the point of emission to an observer) but also by the structure and composition of the disk, as well as the corona.
Table 1 The parameters included in the RELXILL_NK model and their default values. The units of the parameters, where applicable, are indicated. In particular, $r_{\text {in }}$ is specified in units of ISCO by default, but can also be specified in units of $M$

\begin{tabular}{ll}
\hline Parameter & Default value \\
\hline$q_{\text {in }}$ & 3 \\
$q_{\text {out }}$ & 3 \\
$r_{\text {br }}[M]$ & 15 \\
spin & 0.998 \\
$i$ [deg $]$ & 30 \\
$r_{\text {in }}[$ ISCO $]$ & 1 \\
$r_{\text {out }}[M]$ & 400 \\
$\Gamma$ & 2 \\
$\log \xi$ & 3.1 \\
$A_{\text {Fe }}$ & 1 \\
$E_{\text {cut }}[\mathrm{keV}]$ & 300 \\
$R_{f}$ & 3 \\
$\delta$-type & 1 \\
$\delta$-value & 0 \\
$N$ & 1
\end{tabular}

\subsection{The RELXILL_NK model}

Reflection models therefore include parameters from all aspects of the disk-corona model. RELXILL_NK [13, 14,29,30] is a suite of reflection models built for XSPEC that includes a large class of BH-disk-corona models. To illustrate, we describe the eponymous model, RELXILL_NK. Table 1 lists the parameters of the base RELXILL_NK model as well their default values. These parameters account for the different aspects of the system. The disk's emissivity profile is modeled as a simple or broken power law as follows:

$$
\begin{aligned}
& I \propto \frac{1}{r^{q_{\mathrm{in}}}} \quad \text { if } \quad r<r_{\mathrm{br}}, \\
& I \propto \frac{1}{r^{q_{\mathrm{out}}}} \quad \text { if } \quad r \geq r_{\mathrm{br}} .
\end{aligned}
$$

The three free parameters $q_{\mathrm{in}}, q_{\mathrm{out}}$ and $r_{\mathrm{br}}$ form the first three parameters of the RELXILL_NK model. The disk is assumed to be infinitesimally thin, lying in the equatorial plane and composed of particles moving in quasi-geodesic circular orbits. Thus only two disk structure parameters $r_{\text {in }}$ and $r_{\text {out }}$ are needed, to account for the inner and the outer radius of the disk respectively. The elemental constitution of the disk is assumed to follow solar abundances, except iron, which is modeled with $A_{\mathrm{Fe}}$, which is the ratio of iron content in the disk and the iron content in the sun. The ionization of the disk is accounted with $\log \xi$ (where $\xi$ is in units of $\mathrm{erg} \mathrm{cm} / \mathrm{s}$ ), which ranges from 0 (neutral) to 4.7 (highly ionized). The corona is modeled with a power law, whose power law index is given by $\Gamma$ and the high energy cut-off by $E_{\text {cut }}$. The latter is an observational feature and is of the order of the coronal temperature. The $R_{f}$ parameter controls the relative contributions of the coronal and the reflection spectra, and is 
defined as the ratio of intensity emitted towards the disk and that escaping to infinity. The spacetime is modeled using three parameters: the $\mathrm{BH}$ spin $\alpha$, the deformation parameter type $\delta$-type, which can be used to switch between different deformation parameters, and the value of the deformation parameter $\delta$-value. Notably, the BH mass is not a parameter since the reflection spectrum (unlike the thermal spectrum) does not explicitly depend on the BH mass. The observer's viewing angle is modeled with $i$ and finally the strength of the spectrum is accounted with the norm $N$.

\subsection{Numerical method}

The output of the RELXILL_NK model includes the reflection spectrum at the observer. Mathematically, this is given as

$F_{o}\left(v_{o}\right)=\int I_{o}\left(v_{o}, X, Y\right) d \tilde{\Omega}$.

Here $I_{O}$ is the specific intensity (for instance, in units of erg s ${ }^{-1} \mathrm{~cm}^{-2} \mathrm{str}^{-1} \mathrm{~Hz}^{-1}$ ) as detected by an observer. $X$ and $Y$ are the Cartesian coordinates of the image of the disk in the plane of the distant observer, and $d \tilde{\Omega}=d X d Y / D^{2}$ is the element of the solid angle subtended by the image of the disk in the observer's sky. $I_{O}$ can be related to the specific intensity at the point of emission via the Liouville's theorem: $I_{o}=g^{3} I_{e}$, where $g=v_{o} / v_{e}$ is the redshift factor, $v_{o}$ is the photon frequency in the observer's frame at the point of detection, and $v_{e}$ is the photon frequency in the emitter's rest frame at the point of emission. The integration element $d \tilde{\Omega}$ which is presented in terms of variables on the observer plane can also be recast using the redshift factor and the transfer function [57], where the latter is defined as follows:

$f\left(g^{*}, r_{e}, i\right)=\frac{1}{\pi r_{e}} g \sqrt{g^{*}\left(1-g^{*}\right)}\left|\frac{\partial(X, Y)}{\partial\left(g^{*}, r_{e}\right)}\right|$.

Here, $r_{e}$ is the radial coordinate at the point of emission on the disk and $g^{*}$ is the normalized redshift factor, defined as

$g^{*}=\frac{g-g_{\min }}{g_{\max }-g_{\min }}$,

where $g_{\max }=g_{\max }\left(r_{e}, i\right)$ and $g_{\min }=g_{\min }\left(r_{e}, i\right)$ are, respectively, the maximum and the minimum values of the redshift factor $g$ at a fixed $r_{e}$ and for a fixed viewing angle of the observer. The flux can now be rewritten as

$$
\begin{aligned}
F_{o}\left(v_{o}\right)= & \frac{1}{D^{2}} \int_{r_{\text {in }}}^{r_{\text {out }}} \int_{0}^{1} \pi r_{e} \frac{g^{2}}{\sqrt{g^{*}\left(1-g^{*}\right)}} \\
& f\left(g^{*}, r_{e}, i\right) I_{e}\left(v_{e}, r_{e}, \vartheta_{e}\right) d g^{*} d r_{e},
\end{aligned}
$$

where $D$ is the distance of the observer from the source and $\vartheta_{e}$ is the photon's direction relative to the disk at the point of emission. The $r_{e}$-integral ranges from the inner to the outer edge of the disk, and the $g^{*}$-integral ranges from 0 to 1 .

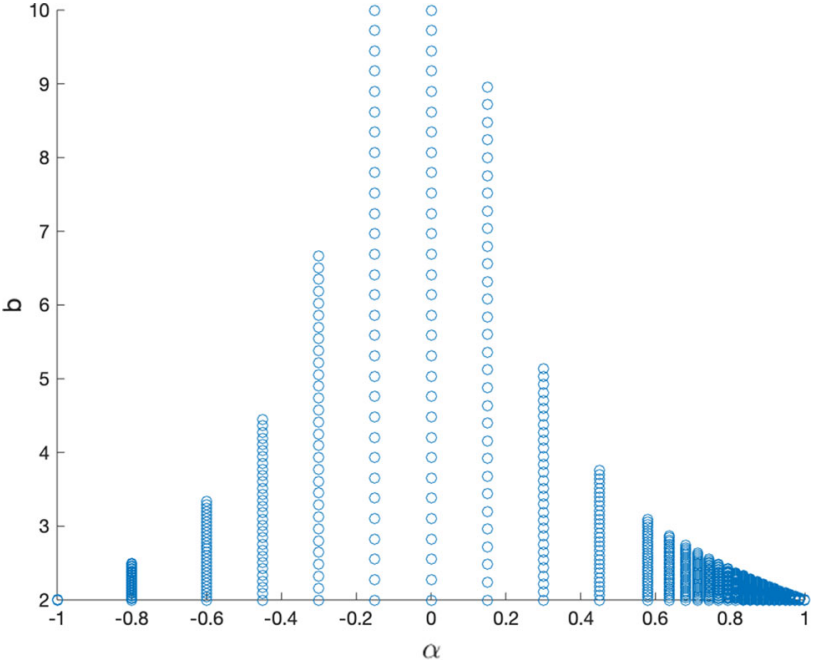

Fig. 2 The grid of values, represented by blue circles, of spin $\alpha$ and deformation parameter $b$ for which the transfer functions are calculated and stored in the FITS table for Case 1. Note that the grid spacings are non-uniform in both $\alpha$ and $b$. See the text for more details

Given the transfer function, the reflection spectrum can be readily calculated using Eq. 16. But it is computationally expensive to calculate the transfer function by tracing photons and using Eq. 14 every time the flux needs to be calculated, therefore RELXILL_NK uses an interpolation scheme to calculate the transfer function for any $\left\{g^{*}, r_{e}, i\right\}$ using a FITS (Flexible Image Transport System) table which stores the transfer functions for some $\left\{g^{*}, r_{e}, i\right\}$. The procedure to create such a table is explained in detail in [13,14]. Here we give a brief overview. The three physical parameters spin $\alpha$, deformation parameter $b$ and the observer's viewing angle $i$, are discretized in a $30 \times 30 \times 22$ grid, respectively. Note that the grid spacing in each dimension is not necessarily uniform, e.g., the grid becomes denser as $\alpha$ increases, since the ISCO radius changes faster with increasing $\alpha$. This scheme enables sufficient resolution during interpolation while maintaining a reasonable table size. The $b$ dimension of the grid depends on the type of deformation parameter under consideration. For the present study, our deformation parameter $b$ is bounded between 2 and $2 /|\alpha|$ in Case 1 (Eq. 11) and between 2 and $4 /|\alpha|-2$ in Case 2 (Eq. 12). We additionally bound $b$ to be below 10. The final $b-\alpha$ grid for Case 1 is shown in Fig. 2 and we have a very similar grid for Case 2 . Note that due to numerical complications, in some cases the bounds on $b$ are more conservative.

At each grid point (i.e., each $\alpha, b$, and $i$ value in the FITS table), the accretion disk is discretized in 100 emission radii $r_{e}$ and 40 equally spaced $g^{*}$ values. ${ }^{4}$ The emission radii grid

\footnotetext{
${ }^{4}$ Because of the way the transfer function is defined in Eq. 14, it goes to zero when the redshift is maximum or minimum, resulting in two branches of transfer function between $g^{*}=0$ and $g^{*}=1$.
} 
ranges from the ISCO to $1000 \mathrm{M}$, and is non-uniform, with higher density near the ISCO. Photons are traced backwards in time from the observer plane to the disk, using the raytracing scheme described in [13,14]. An adaptive algorithm fine-tunes the initial location on the observer plane so that the photon, when back-traced, lands at specific $r_{\mathrm{e}}$ 's. For each such "central" photon, the code calculates the redshift, emission angle, etc. Moreover, four photons closely spaced in the observer plane are launched to calculate the Jacobian and subsequently the transfer function, using Eq. 14. For each $r_{\mathrm{e}}$, about 100 such redshifts, emission angles and transfer functions are calculated, which are then interpolated to get these quantities on the 40 equally spaced values of $g^{*}$, which is stored in the FITS table.

\section{Data analysis}

In this section, we present our analysis of an X-ray observation using the RELXILL_NK model described above.

\subsection{Review}

We chose the source GRS 1915+105 for this analysis. GRS $1915+105$ (or V1487 Aquilae) is a low mass X-ray binary lying 8.6 kiloparsecs away [58]. It features one of the most massive stellar BHs known in our Galaxy. Since its last outburst in 1992, it has been a persistent source of X-rays. In previous work, we have looked at a NUSTAR and a Suzaku observation of this source. In [26], we used RELXILL_NK to analyze a 2012 NUSTAR observation. This observation was difficult to fit and resulted in inconsistent values of the deformation parameter. In a follow up work [27], we used RELXILL_NK to analyze a 2012 Suzaku observation, which required fewer components and resulted in a fit consistent with the Kerr metric. Note that fits to the NUSTAR observation required a thermal component, suggesting a hotter disk, unlike the Suzaku observation where no thermal component was required, suggesting a colder disk. Since the RELXILL_NK model is based on XILLVER which assumes a cold disk, the results of the fits with the Suzaku observation can be expected to be more reliable.

A qualitative picture emerged from previous analyses of GRS 1915+105 thus: the Suzaku observation can be fitted well with the base RELXILL_NK model, the emissivity profile is a broken power law with very high emissivity index in the inner parts of the disk and very small in the outer parts (suggesting a ring-like corona above the accretion disk [59, $60])$, the spin is high $(\sim 0.99)$, the inclination is high $(\sim$ $60-70 \mathrm{deg}$ ) and the spacetime metric is very close to the Kerr metric. Recently, a version of RELXILL_NK developed for thin disks of finite thickness was also used to analyze the Suzaku observation [61], which found that the finite thickness disk version of the RELXILL_NK model provides only a marginally better fit than the base RELXILL_NK model, which assumes infinitesimal thickness.

\subsection{Observations and data reduction}

Suzaku observed GRS 1915+105 for 117 ks on May 7, 2007 (Obs ID 402071010). During this observation, two XIS units were turned off (to preserve telemetry) and a third unit was running in the timing mode, therefore we used data from XIS1 and HXD/PIN instruments only.

The data reduction for this observation has been described in $[26,27]$. We use the same reduced data in the analysis here. In particular, for the XIS1 camera a net exposure time of $28.94 \mathrm{ks}$ (in the $3 \times 3$ editing mode) and for the HXD/PIN a net exposure time of $53.00 \mathrm{ks}$ was achieved. For the analysis, we used the $2.3 \mathrm{keV}$ (since after absorption, there are insufficient photons at low energies for fitting) to $10 \mathrm{keV}$ (to avoid calibration issues near the $\mathrm{Si} \mathrm{K}$ edge) energy band for XIS1 data and 12.0-55.0 keV energy band for HXD/PIN data following [62].

\subsection{Modelling and results}

In our analysis, we employed XSPEC 12.10.1f. Since this observation has been analyzed before with RELXILL_NK, it was natural to guess that the best-fit model combination found previously would also work here. We thus fit the observation with the following model:

MODEL: TBABS*RELXILL_NK, where TBABS describes the galactic absorption [63] and we keep the galactic column density free. The coronal and reflection spectrum are modeled with RELXILL_NK. (The thermal spectrum does not feature in this observation, as shown in [62].) The disk emissivity profile is modeled with a broken power law. The disk inner edge lies at the ISCO, a standard assumption considering during the observation the Eddington scaled accretion luminosity was $20 \%$ $[62,64,65]$, and the outer edge at $400 \mathrm{M}$. The results of the fit for both Case 1 and Case 2 are shown in Table 2. The reduced $\chi^{2}$ is close to 1 , indicating statistical agreement between model and data. The best-fit models and the data to model ratios are presented in Fig. 3. There are no outstanding features that appear unresolved, thus we can be confident that the model fits the data satisfactorily.

We can compare the best-fit parameter values obtained here with their values in other analyses. The emissivity profile, for example, follows previous results with a high $q_{\text {in }}$, nearly zero $q_{\text {out }}$ and the break occurring near $6 M$. The result can be explained within a ring-like corona above the accretion disk $[59,60]$. The spin and inclination are high, as found before. The iron abundance is below solar. Of course, our 
Table 2 Summary of the best-fit values from the analysis of Case 1 and Case 2. The reported uncertainty corresponds to the $90 \%$ confidence level for one relevant parameter. * indicates that the parameter is frozen in the fit. (P) indicates that the $90 \%$ confidence level uncertainty reaches the parameter boundary: $q_{\text {in }}$ and $q_{\text {out }}$ are allowed to vary in the range $[0 ; 10]$, the lower boundary of $A_{\mathrm{Fe}}$ is 0.5 , and the lower boundary of $b$ is 2

\begin{tabular}{lll}
\hline Model & Case 1 & Case 2 \\
\hline TBABS & & \\
$N_{\mathrm{H}} / 10^{22} \mathrm{~cm}^{-2}$ & $8.03_{-0.05}^{+0.08}$ & $8.05_{-0.07}^{+0.12}$ \\
RELXILL_NK & & \\
$q_{\text {in }}$ & $9.9_{-0.5}^{+(\mathrm{P})}$ & $9.80_{-0.82}^{+0.09}$ \\
$q_{\text {out }}$ & $0.00^{+0.16}$ & $0.00^{+0.14}$ \\
$r_{\text {br }}$ & $6.1_{-0.7}^{+0.4}$ & $6.1_{-0.6}^{+0.7}$ \\
$\alpha$ & $0.97_{-0.04}^{+0.02}$ & $0.94_{-0.02}^{+0.05}$ \\
$i$ [deg $]$ & $73.56_{-0.21}^{+1.17}$ & $73.8_{-0.3}^{+1.0}$ \\
$\Gamma$ & $2.208_{-0.024}^{+0.051}$ & $2.210_{-0.020}^{+0.038}$ \\
$\log \xi$ & $2.77_{-0.03}^{+0.03}$ & $2.78_{-0.05}^{+0.06}$ \\
$A_{\mathrm{Fe}}$ & $0.57_{-0.03}^{+0.06}$ & $0.56_{-(\mathrm{P})}^{+0.06}$ \\
$E_{\text {cut }}[\mathrm{keV}]$ & $73_{-4}^{+3}$ & $74_{-3}^{+4}$ \\
$R_{f}$ & $0.50_{-0.04}^{+0.03}$ & $0.50_{-0.05}^{+0.03}$ \\
$b$ & $2.03_{-(\mathrm{P})}^{+0.10}$ & $2.21_{-(\mathrm{P})}^{+0.06}$ \\
$\chi^{2} / d o f$ & $2304.47 / 2208$ & $2303.84 / 2208$ \\
& $=1.04369$ & $=1.04341$ \\
\hline
\end{tabular}

main interest here is the deformation parameter. We find that

$2 \leq b \leq 2.13, \quad 2 \leq c \leq 2.13, \quad$ Case 1

$2 \leq b \leq 2.27, \quad c \equiv 2, \quad$ Case 2

within $90 \%$ uncertainty for one relevant parameter. We can use Eq. 4 to translate these into constraints on the angular momentum and electric and magnetic charges of the black hole as follows:

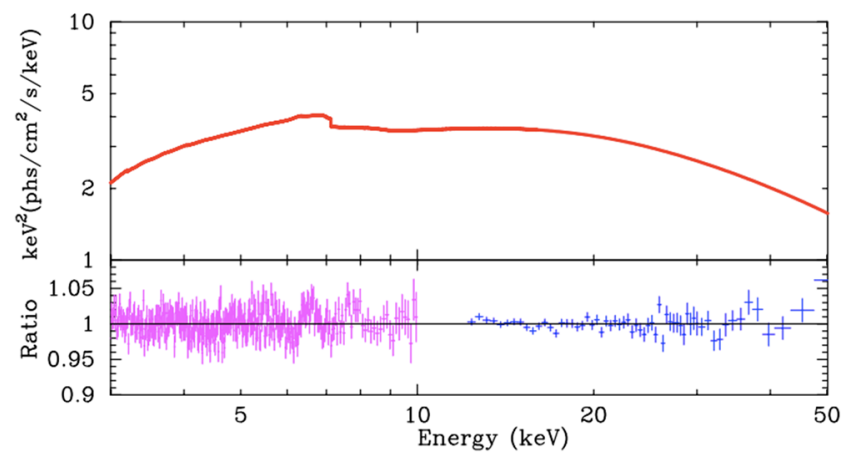

- Case 1:

$$
\begin{gathered}
0.87 \leq J / M^{2} \leq 0.99, \\
0 \leq Q^{2} / M^{2} \leq 0.23, \\
0 \leq P^{2} / M^{2} \leq 0.23 .
\end{gathered}
$$

- Case 2:

$$
\begin{array}{r}
0.86 \leq J / M^{2} \leq 0.99, \\
Q^{2} / M^{2} \equiv 0, \\
0 \leq P^{2} / M^{2} \leq 0.34 .
\end{array}
$$

Since spin and deformation parameter are generally correlated, we also show contour plots of $\alpha$ vs. $b$ in Fig. 4 . The red, green and blue curves are for $68 \%, 90 \%$ and $99 \%$ confidence, respectively, and the gray region indicates parameter space that is excluded according to Eqs. 11 and 12. The correlation between $\alpha$ and $b$ is evident here.

\section{Conclusion}

Alternative theories of gravity have recently become observationally testable in the strong field regime. Among other techniques, X-ray reflection spectroscopy provides one of the strongest constraints on deviations from Einstein's theory. One particularly interesting example of alternatives to general relativity is the Kaluza-Klein theory. In this paper, we use one class of rotating BH solutions of Kaluza-Klein theory described in [40]. We describe the astrophysical system assumed in X-ray spectroscopy analyses and an XSPEC model that calculates the reflection spectrum of this system. The XSPEC model, RELXILL_NK, is then used to analyze a Suzaku observation of the stellar-mass BH system GRS $1915+105$. We review the specific source, describe the specific observation, and use our model to fit the data. We find that the observation is consistent with the Kerr metric, but

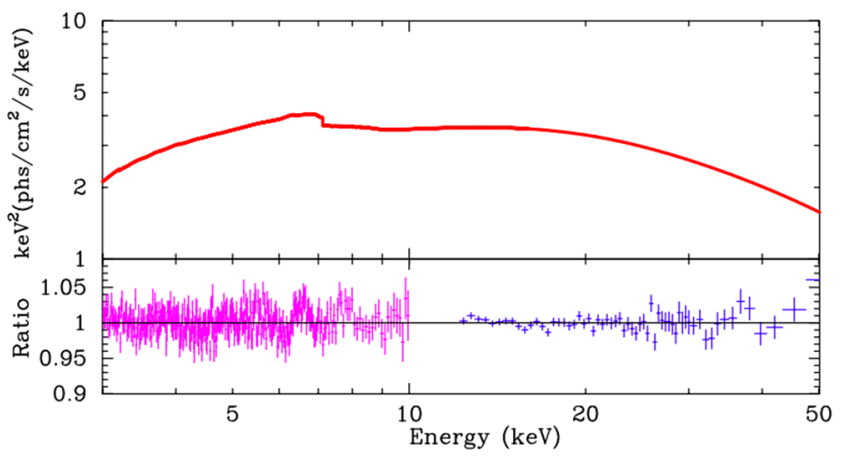

Fig. 3 Best-fit models (upper quadrants) and data-to-best-fit model ratios (bottom quadrants) for Case 1 (left panel) and Case 2 (right panel). In the bottom quadrants, the XIS1 data are in magenta and the HXD/PIN data are in blue. See the text for more details 


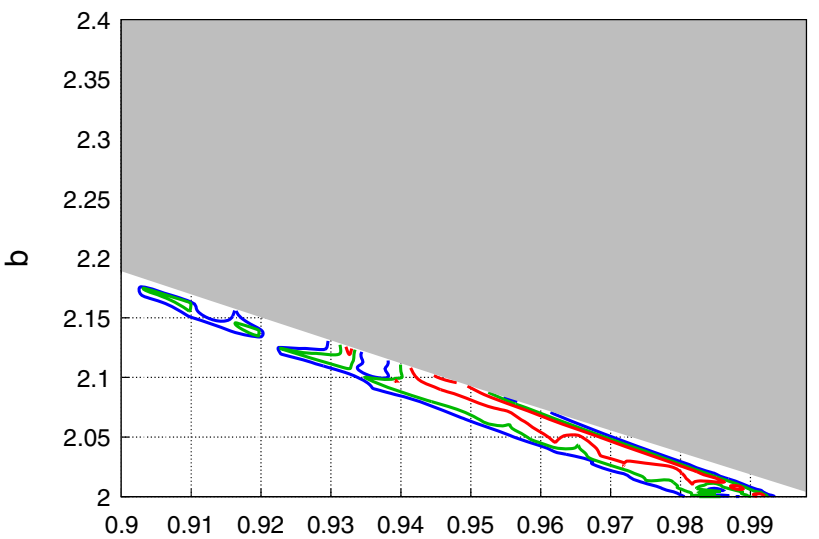

$a_{*}$

Fig. 4 The contour plots of spin $\alpha$ vs $b$ for Case 1 (left panel) and Case 2 (right panel). The red, green and blue lines show the 68\%, 90\%, and $99 \%$ confidence region boundaries respectively. The greyed region

allows for some deviation away from it. It will be interesting to test for signatures of such deviations in other astrophysical sources in future.

Acknowledgements This work was supported by the Innovation Program of the Shanghai Municipal Education Commission, Grant No. 2019-01-07-00-07-E00035, and the National Natural Science Foundation of China (NSFC), Grant No. 11973019. A.B.A. acknowledges the support from the Shanghai Government Scholarship (SGS). S.N. acknowledges support from the Excellence Initiative at Eberhard-Karls Universität Tübingen and the Alexander von Humboldt Foundation.

Data Availability Statement This manuscript has no associated data or the data will not be deposited. [Authors' comment: We analyzed publicly available data that can be downloaded for free, for example, from the HEASARC website https://heasarc.gsfc.nasa.gov/.]

Open Access This article is licensed under a Creative Commons Attribution 4.0 International License, which permits use, sharing, adaptation, distribution and reproduction in any medium or format, as long as you give appropriate credit to the original author(s) and the source, provide a link to the Creative Commons licence, and indicate if changes were made. The images or other third party material in this article are included in the article's Creative Commons licence, unless indicated otherwise in a credit line to the material. If material is not included in the article's Creative Commons licence and your intended use is not permitted by statutory regulation or exceeds the permitted use, you will need to obtain permission directly from the copyright holder. To view a copy of this licence, visit http://creativecomm ons.org/licenses/by/4.0/.

Funded by SCOAP ${ }^{3}$.

\section{References}

1. C.M. Will, Liv. Rev. Rel. 17, 4 (2014). https://doi.org/10.12942/ lrr-2014-4. arXiv:1403.7377 [gr-qc]

2. C. Bambi, Rev. Mod. Phys. 89, 025001 (2017). https://doi.org/10. 1103/RevModPhys.89.025001. arXiv:1509.03884 [gr-qc]

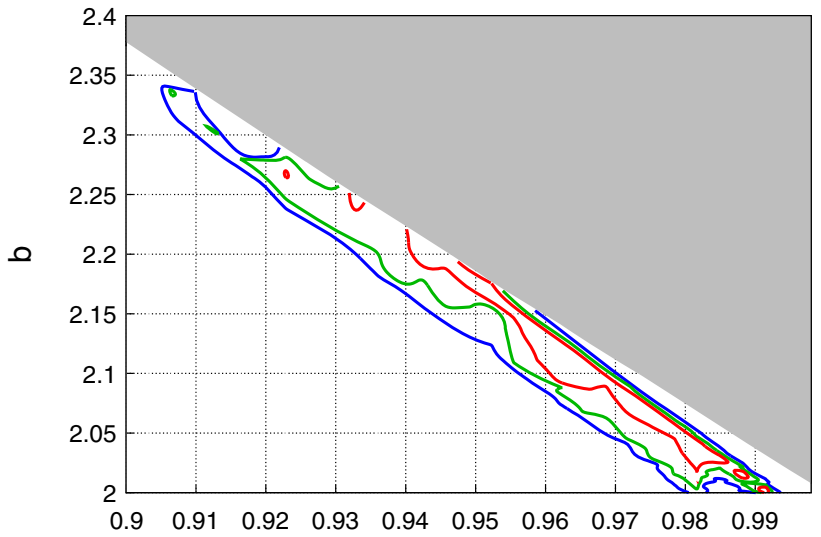

$a_{*}$

is pathological because outside of the parameter space described by Eqs. 11 and 12, respectively, and is excluded from the analysis. See the text for more details

3. C. Bambi, Black Holes: A Laboratory for Testing Strong Gravity (Springer, Berlin, 2017). https://doi.org/10.1007/ 978-981-10-4524-0

4. B. Abbott et al. (LIGO Scientific, Virgo), Phys. Rev. D 100, 104036 (2019). https://doi.org/10.1103/PhysRevD.100.104036. arXiv: 1903.04467 [gr-qc]

5. Y. Mizuno, Z. Younsi, C.M. Fromm, O. Porth, M. De Laurentis, H. Olivares, H. Falcke, M. Kramer, L. Rezzolla, Nat. Astron. 2, 585 (2018). https://doi.org/10.1038/ s41550-018-0449-5. arXiv:1804.05812 [astro-ph.GA]

6. A. Cardenas-Avendano, S. Nampalliwar, N. Yunes, (2019). arXiv: 1912.08062 [gr-qc]

7. C. Bambi, JCAP 03, 034 (2014). https://doi.org/10.1088/ 1475-7516/2014/03/034. arXiv:1308.2470 [gr-qc]

8. C. Li, S.-F. Yan, L. Xue, X. Ren, Y.-F. Cai, D.A. Easson, Y.-F. Yuan, H. Zhao, (2019). arXiv:1912.12629 [astro-ph.CO]

9. C.S. Reynolds, Space Sci. Rev. 183, 277 (2014). https://doi.org/ 10.1007/s11214-013-0006-6. arXiv:1302.3260 [astro-ph.HE]

10. C. Bambi, A.B. Abdikamalov, D. Ayzenberg, Z. Cao, H. Liu, S. Nampalliwar, A. Tripathi, J. Wang-Ji, Y. Xu, Universe 4, 79 (2018). https://doi.org/10.3390/universe4070079. arXiv:1806.02141 [gr$\mathrm{qc}]$

11. J. García et al., Astrophys. J. 782, 76 (2014). https://doi.org/10. 1088/0004-637X/782/2/76. arXiv:1312.3231 [astro-ph.HE]

12. T. Dauser et al., Mon. Not. R. Astron. Soc. 444, 100 (2014). https:// doi.org/10.1093/mnrasl/slu125. arXiv:1408.2347 [astro-ph.HE]

13. C. Bambi et al., Astrophys. J. 842, 76 (2017). https://doi.org/10. 3847/1538-4357/aa74c0. arXiv:1607.00596 [gr-qc]

14. A.B. Abdikamalov, D. Ayzenberg, C. Bambi, T. Dauser, J.A. Garcia, S. Nampalliwar, Astrophys. J. 878, 91 (2019). https://doi.org/ 10.3847/1538-4357/ab1f89. arXiv:1902.09665 [gr-qc]

15. S. Nampalliwar, S. Xin, S. Srivastava, A.B. Abdikamalov, D. Ayzenberg, C. Bambi, T. Dauser, J.A. Garcia, A. Tripathi, (2019). arXiv: 1903.12119 [gr-qc]

16. H. Liu, A.B. Abdikamalov, D. Ayzenberg, C. Bambi, T. Dauser, J.A. Garcia, S. Nampalliwar, Phys. Rev. D 99, 123007 (2019). https:// doi.org/10.1103/PhysRevD.99.123007. arXiv:1904.08027 [gr-qc]

17. B. Zhou, A. Tripathi, A.B. Abdikamalov, D. Ayzenberg, C. Bambi, S. Nampalliwar, M. Zhou, (2019). arXiv:1908.05177 [gr-qc]

18. Z. Cao, S. Nampalliwar, C. Bambi, T. Dauser, J.A. Garcia, Phys. Rev. Lett. 120, 051101 (2018). https://doi.org/10.1103/ PhysRevLett.120.051101. arXiv:1709.00219 [gr-qc] 
19. A. Tripathi, S. Nampalliwar, A.B. Abdikamalov, D. Ayzenberg, J. Jiang, C. Bambi, Phys. Rev. D 98, 023018 (2018). https://doi.org/ 10.1103/PhysRevD.98.023018. arXiv:1804.10380 [gr-qc]

20. Y. Xu, S. Nampalliwar, A.B. Abdikamalov, D. Ayzenberg, C. Bambi, T. Dauser, J.A. Garcia, J. Jiang, Astrophys. J. 865, 134 (2018). https://doi.org/10.3847/1538-4357/aadb9d. arXiv: 1807.10243 [gr-qc]

21. K. Choudhury, S. Nampalliwar, A.B. Abdikamalov, D. Ayzenberg, C. Bambi, T. Dauser, J.A. Garcia, Astrophys. J. 879, 80 (2019). https://doi.org/10.3847/1538-4357/ab24d6. arXiv:1809.06669 [gr-qc]

22. M. Zhou, Z. Cao, A. Abdikamalov, D. Ayzenberg, C. Bambi, L. Modesto, S. Nampalliwar, Phys. Rev. D 98, 024007 (2018). https:// doi.org/10.1103/PhysRevD.98.024007. arXiv:1803.07849 [gr-qc]

23. M. Zhou, A. Abdikamalov, D. Ayzenberg, C. Bambi, L. Modesto, S. Nampalliwar, Y. Xu, EPL 125, 30002 (2019). https://doi.org/10. 1209/0295-5075/125/30002

24. A. Tripathi, S. Nampalliwar, A.B. Abdikamalov, D. Ayzenberg, C. Bambi, T. Dauser, J.A. Garcia, A. Marinucci, Astrophys. J. 875, 56 (2019). https://doi.org/10.3847/1538-4357/ab0e7e. arXiv: 1811.08148 [gr-qc]

25. A. Tripathi et al., Astrophys. J. 874, 135 (2019). https://doi.org/10. 3847/1538-4357/ab0a00. arXiv:1901.03064 [gr-qc]

26. Y. Zhang, A.B. Abdikamalov, D. Ayzenberg, C. Bambi, T. Dauser, J.A. Garcia, S. Nampalliwar, Astrophys. J. 875, 41 (2019). https:// doi.org/10.3847/1538-4357/ab0e79. arXiv:1901.06117 [gr-qc]

27. Y. Zhang, A.B. Abdikamalov, D. Ayzenberg, C. Bambi, S. Nampalliwar, Astrophys. J. 884, 147 (2019). https://doi.org/10.3847/ 1538-4357/ab4271. arXiv:1907.03084 [gr-qc]

28. A. Tripathi, A.B. Abdikamalov, D. Ayzenberg, C. Bambi, S. Nampalliwar, Phys. Rev. D 99, 083001 (2019). https://doi.org/10.1103/ PhysRevD.99.083001. arXiv:1903.04071 [gr-qc]

29. “RELXILL_NK," http://www.tat.physik.uni-tuebingen.de/ nampalliwar/relxill_nk/ ()

30. “RELXILL_NK," http://www.physics.fudan.edu.cn/tps/people/ bambi/Site/RELXILL_NK.html ()

31. M. Rizwan, M. Jamil, K. Jusufi, Phys. Rev. D 99, 024050 (2019). https://doi.org/10.1103/PhysRevD.99.024050. arXiv:1812.01331 [gr-qc]

32. M. Rizwan, M. Jamil, A. Wang, Phys. Rev. D 98, 024015 (2018), [Erratum: Phys.Rev.D 100, 029902 (2019)], https://doi.org/10. 1103/PhysRevD.98.024015. arXiv:1802.04301 [gr-qc]

33. S. Haroon, M. Jamil, K. Lin, P. Pavlovic, M. Sossich, A. Wang, Eur. Phys. J. C 78, 519 (2018). https://doi.org/10.1140/epjc/ s10052-018-5986-7. arXiv:1712.08762 [gr-qc]

34. C. Chakraborty, M. Patil, P. Kocherlakota, S. Bhattacharyya, P.S. Joshi, A. Królak, Phys. Rev. D 95, 084024 (2017). https://doi.org/ 10.1103/PhysRevD.95.084024. arXiv:1611.08808 [gr-qc]

35. V. Khachatryan et al., (CMS) Phys. Lett. B 697, 434 (2011). https://doi.org/10.1016/j.physletb.2011.02.032. arXiv:1012.3375 [hep-ex]

36. P.S. Wesson, J. Ponce de Leon, Astron. Astrophys. 294, 1 (1995)

37. L. Amarilla, E.F. Eiroa, Phys. Rev. D 87, 044057 (2013). https:// doi.org/10.1103/PhysRevD.87.044057. arXiv:1301.0532 [gr-qc]

38. V. Cardoso, L. Gualtieri, C.J. Moore, Phys. Rev. D 100, 124037 (2019). https://doi.org/10.1103/PhysRevD.100.124037. arXiv: 1910.09557 [gr-qc]

39. D. Andriot, G. Lucena Gómez, JCAP 06, 048 (2017), [Erratum: JCAP 05, E01 (2019)], https://doi.org/10.1088/1475-7516/2017/ 06/048. arXiv:1704.07392 [hep-th]

40. M. Azreg-Aïnou, M. Jamil, K. Lin, Chin. Phys. C 44, 065101 (2020). https://doi.org/10.1088/1674-1137/44/6/065101. arXiv:1907.01394 [gr-qc]

41. G.T. Horowitz, T. Wiseman, "General black holes in Kaluza-Klein theory," in Black holes in higher dimensions (2012) pp. 69-98, arXiv:1107.5563 [gr-qc]
42. P. Dobiasch, D. Maison, Gen. Rel. Gravit. 14, 231 (1982). https:// doi.org/10.1007/BF00756059

43. A. Chodos, S.L. Detweiler, Gen. Rel. Gravit. 14, 879 (1982). https://doi.org/10.1007/BF00756803

44. G. Gibbons, D. Wiltshire, Annals Phys. 167, 201 (1986), [Erratum: Annals Phys. 176, 393 (1987)] https://doi.org/10.1016/ S0003-4916(86)80012-4

45. F. Larsen, Nucl. Phys. B 575, 211 (2000). https://doi.org/10.1016/ S0550-3213(00)00064-X. arXiv:hep-th/9909102

46. D. Rasheed, Nucl. Phys. B 454, 379 (1995). https://doi.org/10. 1016/0550-3213(95)00396-A. arXiv:hep-th/9505038

47. T. Matos, C. Mora, Class. Quantum Gravit. 14, 2331 (1997). https:// doi.org/10.1088/0264-9381/14/8/027. arXiv:hep-th/9610013

48. H. Ishihara, K. Matsuno, Prog. Theor. Phys. 116, 417 (2006). https://doi.org/10.1143/PTP.116.417. arXiv:hep-th/0510094

49. T. Wang, Nucl. Phys. B 756, 86 (2006). https://doi.org/10.1016/j. nuclphysb.2006.09.001. arXiv:hep-th/0605048

50. J. Park, Class. Quantum Gravit. 15, 775 (1998). https://doi.org/10. 1088/0264-9381/15/4/006. arXiv:hep-th/9503084

51. J. Overduin, P. Wesson, Phys. Rep. 283, 303 (1997). https://doi. org/10.1016/S0370-1573(96)00046-4. arXiv:gr-qc/9805018

52. M. Allahverdizadeh, K. Matsuno, Phys. Rev. D 81, 044001 (2010). https://doi.org/10.1103/PhysRevD.81.044001. arXiv:0908.2484 [hep-th]

53. J.H. Horne, G.T. Horowitz, Phys. Rev. D 46, 1340 (1992). https:// doi.org/10.1103/PhysRevD.46.1340. arXiv:hep-th/9203083

54. D. Stojkovic, K. Freese, Phys. Lett. B 606, 251 (2005). https://doi. org/10.1016/j.physletb.2004.12.019. arXiv:hep-ph/0403248

55. S.L. Liebling, C. Palenzuela, Phys. Rev. D 94, 064046 (2016). https://doi.org/10.1103/PhysRevD.94.064046. arXiv:1607.02140 [gr-qc]

56. I.D. Novikov, K.S. Thorne, in Proceedings, Ecole d'Eté de Physique Théorique: Les Astres Occlus: Les Houches, France, August, 1972 (1973), pp. 343-550

57. C.T. Cunningham, Astrophys. J. 202, 788 (1975). https://doi.org/ $10.1086 / 154033$

58. M. Reid, J. McClintock, J. Steiner, D. Steeghs, R. Remillard, V. Dhawan, R. Narayan, Astrophys. J. 796, 2 (2014). https://doi.org/ 10.1088/0004-637X/796/1/2. arXiv:1409.2453 [astro-ph.GA]

59. G. Miniutti, A. Fabian, R. Goyder, A. Lasenby, Mon. Not. R. Astron. Soc. 344, L22 (2003). https://doi.org/10.1046/j. 1365-8711.2003.06988.x. arXiv:astro-ph/0307163

60. D. Wilkins, A. Fabian, Mon. Not. R. Astron. Soc. 414, 1269 (2011). https://doi.org/10.1111/j.1365-2966.2011.18458.x. arXiv: 1102.0433 [astro-ph.HE]

61. A.B. Abdikamalov, D. Ayzenberg, C. Bambi, T. Dauser, J.A. Garcia, S. Nampalliwar, A. Tripathi, M. Zhou, (2020), arXiv:2003.09663 [astro-ph.HE]

62. J. Blum, J. Miller, A. Fabian, M. Miller, J. Homan, M. van der Klis, E. Cackett, R. Reis, Astrophys. J. 706, 60 (2009). https://doi.org/ 10.1088/0004-637X/706/1/60. arXiv:0909.5383 [astro-ph.HE]

63. J. Wilms, A. Allen, R. McCray, Astrophys. J. 542, 914 (2000). https://doi.org/10.1086/317016. arXiv:astro-ph/0008425 [astro$\mathrm{ph}]$

64. J.F. Steiner, J.E. McClintock, R.A. Remillard, L. Gou, S. Yamada, R. Narayan, Astrophys. J. 718, L117 (2010). https://doi.org/10. 1088/2041-8205/718/2/L117. arXiv:1006.5729 [astro-ph.HE]

65. A.K. Kulkarni, R.F. Penna, R.V. Shcherbakov, J.F. Steiner, R. Narayan, A. Sadowski, Y. Zhu, J.E. McClintock, S.W. Davis, J.C. McKinney, Mon. Not. R. Astron. Soc. 414, 1183 (2011). https://doi. org/10.1111/j.1365-2966.2011.18446.x. arXiv:1102.0010 [astroph.HE] 